\title{
The Adaptogens Rhodiola and Schizandra Modify the Response to Immobilization Stress in Rabbits by Suppressing the Increase of Phosphorylated Stress-activated Protein Kinase, Nitric Oxide and Cortisol
}

\author{
Alexander Panossian ${ }^{1}$, Marina Hambardzumyan ${ }^{2}$, Areg Hovhanissyan ${ }^{2}$ \\ and Georg Wikman ${ }^{1}$ \\ ${ }_{1}^{1}$ Swedish Herbal Institute Research and Development, Spårvägen 2, Åsklöster 43296, Sweden. \\ 2 "ExLab" Expert Analytical Laboratory of Armenia Drug Agency, Komitas Ave. 49/4, 375051 Yerevan, \\ Armenia.
}

\begin{abstract}
Adaptogens possess anti-fatigue and anti-stress activities that can increase mental and physical working performance against a background of fatigue or stress. The aim of the present study was to ascertain which mediators of stress response are significantly involved in the mechanisms of action of adaptogens, and to determine their relevance as biochemical markers for evaluating anti-stress effects in rabbits subjected to restraint stress. Blood levels of stress-activated protein kinase (SAPK/JNK), the phosphorylated kinase p-SAPK/p-JNK, nitric oxide (NO), cortisol, testosterone, prostaglandin $\mathrm{E}_{2}$, leukotriene $\mathrm{B}_{4}$ and thromboxane $\mathrm{B}_{2}$ were determined in groups of animals prior to daily oral administration of placebo, rhodioloside or extracts of Eleutherococcus senticosus, Schizandra chinensis, Rhodiola rosea, Bryonia alba and Panax ginseng over a 7 day period. Ten minutes after the final treatment, animals were immobilized for 2 hours and blood levels of the markers re-determined. In the placebo group, only p-SAPK/p-JNK, NO and cortisol were increased significantly (by 200-300\% cf basal levels) following restraint stress, whilst in animals that had received multiple doses of adaptogens/stress-protectors, the levels of NO and cortisol remained practically unchanged after acute stress. Rhodioloside and extracts of $S$. chinensis and $R$. rosea were the most active inhibitors of stress-induced p-SAPK/p-JNK. E. senticosus, B. alba and $P$. ginseng exerted little effect on $\mathrm{p}-\mathrm{SAPK} / \mathrm{p}-\mathrm{JNK}$ levels. It is suggested that the inhibitory effects of $R$. rosea and $S$. chinensis on $\mathrm{p}-\mathrm{SAPK} / \mathrm{p}-\mathrm{JNK}$ activation may be associated with their anti-depressant activity as well as their positive effects on mental performance under stress.
\end{abstract}

Keywords: Adaptogens, stress, Rhodiola rosea, Schizandra chinensis, rhodioloside, p-SAPK/p-JNK, nitric oxide, cortisol.

\section{Introduction}

The term "adaptogen" was coined in the middle of the 20th century by the Russian scientist Lazarev (Brekhman and Dardymov, 1968) to describe medicinal plants that are able to enhance the so-called "state of non-specific resistance" of an organism to stress. It is now accepted that true adaptogens must: (i) possess stress-protective effects (i.e. reduction of stress-induced damage) such as anti-fatigue, antiinfection and restorative activities; (ii) present stimulating effects, following both single and multiple administration, that give rise to an increase in working capacity and mental performance against a background of fatigue and stress (such stimulating effects must be different from those of conventional CNS stimulants and anabolics that deplete energy and plastic resources of the organism and are accompanied by negative side effects including drug withdrawal syndrome); and (iii) be innocuous and not disturb the normal level of body functions, but rather present a normalizing influence on the pathologic state, independent of the nature of that state (Brekhman and Dardymov, 1968). It should be noted that only Schizandra chinensis, Eleutherococcus senticosus and Rhodiola rosea have been found to be fully compliant with this specific definition of adaptogen (Panossian and Wagner, 2005).

Whilst the concept of an adaptogen is readily understood from the physiological standpoint, it is not so easy to accept pharmacologically when it is necessary to define the mechanism of action of a medicine and to formulate indications of its use. In particular, the stress-protective effect of an adaptogen results from the adaptation of the organism to repeated stimulating effects of the drug (Brekhman and

Correspondence: A. Panossian, Swedish Herbal Institute Research and Development, Spårvägen 2, Åsklöster 43296, Sweden. Tel / Fax: (46) 430-23723; Email: alexander.panossian@shi.se

Please note that this article may not be used for commercial purposes. For further information please refer to the copyright statement at http://www.la-press.com/copyright.htm 
Dardymov, 1968; Wagner et al. 1994; Panossian et al. 1999a; Panossian, 2003; Panossian and Wagner, 2005). Since adaptation to stress is associated with the interactions of numerous mediators of the nervous, endocrine and immune systems, and is regulated at all levels of organization (cellular, regulating systems, whole organism) (Fink, 2000), it is very unlikely that different stressprotectors have the same mechanism of action.

The active components of stress-protective plants and adaptogens (Table 1) can be formally divided into three main groups, namely, tetra(penta)cyclic terpenoids, phenyl- and phenylethyl-propanoids and derivatives, and oxylipins. On the basis of the chemical nature of their active principles, some indication of the possible mechanism of action of these plants may be derived. Thus, the extracts of Panax ginseng, Withania somnifera, Bryonia alba and Aralia mandshurica contain phytosterols, and tetracyclic and pentacyclic triterpenes, that likely exert their effect on the hypothalamus-pituitary-adrenal (HPA) axis in which cortisone plays a key role during stress. Typically these adaptogens prevent or at least decrease certain hormonal changes, such as the increased level of cortisone, that are characteristic of a stress reaction (Panossian et al. 1999b; Kim et al. 2003a). Plants such as R. rosea and $S$. chinensis accumulate phenolic secondary metabolites such as phenyl- and phenylethylpropanoids and their dimeric lignans (Wagner et al. 1996; Saratikov and Krasnov 2004). Such compounds can play an active role in stress response in respect of achieving a state of maximum work capacity as would be required in fight-orflight situations (Lüllmann et al. 2005). Interestingly, E. senticosus contains both types of biologically active, low molecular weight compound and exhibits a very wide range of pharmacological effects (World Health Organization, 2002).

The pharmacological assessment of adaptogens typically includes evaluation of their stimulating, tonic and stress-protective activities in model systems in which animals are subjected to various stress conditions (Panossian and Wikman, 2005). Despite considerable research effort, however, it still remains somewhat unclear which mediators of stress response are predominantly involved in

Table 1. Classes of secondary metabolites identified in Panax ginseng, Bryonia alba, Withania somnifera, Aralia mandshurica, Rhodiola rosea, Schizandra chinensis and Eleutherococcus senticosus.

Group I: Stress-protectors

Panax ginseng

Tetracyclic triterpenes and their glycosides

Pentacyclic triterpenes and their glycosides

Polyacetylenes

\section{Bryonia alba}

Tetracyclic triterpenes and their glycosides

Pentacyclic triterpenes

Sterols and their glycosides

Oxylipins and glycolipids

\section{Withania somnifera}

Tetracyclic triterpene lactones and their glycosides

Sterols and their glycosides

Alkaloids

\section{Aralia mandshurica}

Pentacyclic triterpenes and their glycosides

\section{Group II: Adaptogens}

\section{Rhodiola rosea}

Phenylethyl glycosides

Phenylpropanoids

Flavonoids

Phenolics

Polyphenolics

Lignans

Flavolignans

\section{Schizandra chinensis}

Dibenzo[a,c]cyclooctadiene

Sterols

Organic and fatty acids,

Vitamins A,C and E

Sterols

\section{Eleutherococcus senticosus}

Pentacyclic and tetracyclic triterpene glycosides

Sterols

Phenylpropanoids

Lignans

Polysaccharides (heteroglycans, eleutherans)

Coumarins 
the mechanisms of action of adaptogens, and which biochemical markers need to be assayed in the evaluation of drug efficacy.

In order to address this problem further, we have determined blood levels of the potential stress response markers (Fink, 2000) stress-activated protein kinase/Jun N-terminal protein kinase (SAPK/JNK), the phosphorylated kinase p-SAPK/ p-JNK, NO, cortisol, testosterone, prostaglandin $\mathrm{E}_{2}$, leukotriene $\mathrm{B}_{4}$ and thromboxane $\mathrm{B}_{2}$ in laboratory rabbits, treated with stress-protectors and adaptogens, both before and after immobilization stress. The most extensively studied stressprotectors, $B$. alba and $P$. ginseng, and the adaptogens, E. senticosus, $R$. rosea, and $S$. chinensis (Panossian et al. 1997; Upton, 1999; World Health Organization, 1999, 2002; Saratikov and Krasnov, 2004), together with rhodioloside, an active ingredient of $R$. rosea (Aksenova et al. 1968), were employed in this study.

\section{Materials and Methods}

Details of the project were submitted to and approved by the Ethics Committee of the Armenian Drug and Medical Technology Agency of the Ministry of Health of the Republic of Armenia. The principles of laboratory animal care, as delineated in EEC Directive 75/318 (1994), were followed throughout the study.

\section{Study animals}

Male Chinchilla rabbits were obtained from the breeding unit of the Institute of Fine Organic Chemistry of the National Academy of Science, Yerevan, Armenia. All animals were clinically examined upon arrival and any that showed signs of abnormality or disease were excluded. The 39 animals employed in the study were maintained in the animal house under a $12 \mathrm{~h}$ light / dark cycle for 10-15 days prior to the commencement of the study and were offered standard rat chow ad libitum. Any animals considered to be unsuitable were replaced before the start of the study, and no animals were replaced after the study had commenced. The weights of the study animals immediately prior to the commencement of the study were in the range $2.5-3.0 \mathrm{~kg}$.

During the study period, animals were kept separately in cages $(150 \times 100 \times 100 \mathrm{~cm})$ consisting of polystyrene cases and lattice framed steel lids: wood-sawdust was used as bedding. The target ranges for the temperature and the relative humidity of the animal house were $22 \pm 4^{\circ} \mathrm{C}$ and $40 \pm 5 \%$, respectively, and the air was changed 1-2 times / $\mathrm{h}$. Throughout the study, a standardized diet (Combi/ Yerevan Combi-Corm Plant) was provided, but feeding was discontinued prior to the administration of a test material. Only drinking water was offered ad libitum.

\section{Plant extracts}

Extracts of E. senticosus roots (SHE-3, batch Ex 20729 standardized for the content of eleutherosides E and B), R. rosea roots (SHR-5, batch Ex 20715 standardized for the content of rhodioloside, tyrosole, triandrin and rosavin) and $S$. chinensis berries (SHS-2, batch Ex 20646 standardized for the content of schizandrin and $\gamma$-schizandrin) were supplied by the Swedish Herbal Institute (Gothenburg, Sweden). Extracts of B. alba roots (batch 026 containing $4 \% \mathrm{w} / \mathrm{w}$ of total cucurbitacins expressed as cucurbitacin $\mathrm{R}$ equivalents) and of $P$. ginseng roots (containing $10.5 \% \mathrm{w} / \mathrm{w}$ of ginsenosides) were prepared by extraction of the herbal material with $70 \%$ ethanol and manufactured using commonly employed commercial processes. Rhodoloside (syn. salidroside; batch S10402) was obtained from Vilar (Moscow, Russia).

\section{Dosage regimes and immobilization of study animals}

The doses employed for the six different treatments involved in this study were: $E$. senticosus root extract $-6.5 \mathrm{mg} / \mathrm{kg}, R$. rosea root extract $-1 \mathrm{mg} / \mathrm{kg}, S$. chinensis berry extract $-22 \mathrm{mg} / \mathrm{kg}$; $B$. alba root extract $-15 \mathrm{mg} / \mathrm{kg}, P$. ginseng root extract $-6 \mathrm{mg} / \mathrm{kg}$, and rhodioloside $-0.5 \mathrm{mg} / \mathrm{kg}$. In each case, a suspension of the test material was prepared freshly each day by shaking an appropriate amount in distilled water such that the a $10 \mathrm{ml}$ volume of the final suspension contained the stated dosage amount per kg body weight. Starting on day 2, study animals were treated each day (during the period 10.00-10.30) for 7 consecutive days with an appropriate volume of suspension $(10 \mathrm{ml} / \mathrm{kg})$, which was shaken gently immediately prior to administration and delivered by oral gavage. Equivalent volumes of distilled water were supplied to placebo animals during the same period.

The study animals were divided into three groups and treated as follows: those in group A (3 animals) were treated with distilled water for 7 
days and subjected to forced immobilization on days 2 and 8; those in group B (3 animals in each of 6 different treatment sub-groups) were treated with a study drug for 7 days but were not subjected to immobilization; and those in group C ( 3 animals in each of 6 different treatment sub-groups) were treated with a study drug for 7 days and subjected to forced immobilization on days 2 and 8. Immobilization, which was conducted $10 \mathrm{~min}$ after the administration of drug or placebo, was carried out by fixing the head and pads of the rabbit to a $1.1 \times 0.4 \mathrm{~m}$ plate and maintaining the animal in this state for $2 \mathrm{~h}$ without food and water.

\section{Blood sampling}

A $10 \mathrm{ml}$ sample of blood was collected from each rabbit on day 1 of the study (i.e. the day prior to the commencement of drug administration). Blood samples were collected from the heart cavity under aseptic conditions by inserting the needle of a $20 \mathrm{ml}$ syringe into the 3 rd intercostal space at a location 3-4 $\mathrm{mm}$ from the left hand end of the sternum. Over the following $5 \mathrm{~min}$ period, a $20 \mathrm{ml}$ volume of warm sterile saline solution was administered subcutaneously and the animal was transferred to a standard cage and given free access to food and water. For rabbits receiving adaptogens/stress-protectors, blood samples $(10 \mathrm{ml})$ were similarly collected on day 2 either immediately after immobilization (group C) or at the same designated time (group B). On day 8 of the study, blood samples were collected in a similar manner from all animals, either immediately after immobilization (groups A and C) or at the same designated time (group B).

Plasma was obtained by transferring a portion of the freshly collected blood sample into a $4 \mathrm{ml}$ sterile heparinised (lithium heparin) Vacuette ${ }^{\circledR}$ tube (Greiner Bio-one $\mathrm{GmbH}$, Kremsmuenster, Austria) and centrifuging at $600 \mathrm{~g}$ for $15 \mathrm{~min}$. The remaining portion of the blood sample was allowed to clot at room temperature in the original plain collecting tube, and serum was separated by centrifugation in a micro-centrifuge at $3000 \mathrm{rpm}$ for $10 \mathrm{~min}$. Plasma and serum samples were stored at $-40^{\circ} \mathrm{C}$ until required for assay.

\section{Biochemical assays}

\section{p-JNK1/2}

Assays were performed using Phospho JNK1 Colorimetric (EIA) TiterZyme ${ }^{\circledR}$ Kits (Assay Designs,
Ann Arbor, MI, U.S.A.; product number 900-106) containing mouse monoclonal antibody specific to JNK immobilized on a microtitre plate. Blood plasma $(100 \mu \mathrm{l})$ was mixed with $0.9 \mathrm{ml}$ of RIPA cell lysis buffer [50 mM Tris $\mathrm{HCl}(\mathrm{pH} 7.4), 150 \mathrm{mM}$ $\mathrm{NaCl}, 1$ mM EDTA, 1 mM EGTA, 1\% Triton X-100, $1 \%$ sodium deoxycholate and $0.1 \%$ SDS] containing $0.5 \mathrm{ml} / 1$ protease inhibitor cocktail (PIC; Sigma, St. Louis, MO, U.S.A.), $1 \mathrm{mM}$ phenylmethylsulfonyl fluoride (PMSF), $2 \mathrm{mM}$ sodium orthovanadate and $20 \mathrm{mM}$ sodium pyrophosphate, and mixed thoroughly for $5 \mathrm{~min}$. The test solution was prepared by diluting $1 \mathrm{ml}$ of this mixture with $9 \mathrm{ml}$ of assay buffer solution (MOPSO buffered saline containing proteins, detergents, phosphatase inhibitor, $0.5 \mathrm{ml} / 1$ PIC and $1 \mathrm{mM}$ PMSF). Aliquots $(100 \mu \mathrm{l})$ of test solutions, or reference standards, were pipetted in duplicate into wells of the microtitre plate and incubated at room temperature for $1 \mathrm{~h}$. Wells were washed with Tris buffered saline containing detergents, and $100 \mu$ l of biotinylated mouse monoclonal antibody to $\mathrm{p}$-JNK was added to each well in order to bind the immobilized analyte. After a further incubation of $1 \mathrm{~h}$, excess antibody was washed out and streptavidin conjugated to horseradish peroxidase was added (except to the blank) to bind to the biotinylated monoclonal p-JNK antibody. Following a $30 \mathrm{~min}$ incubation, excess conjugate was washed out, $100 \mu \mathrm{l}$ of substrate solution $\left(3,3,3,5,5^{\prime}\right.$ tetramethylbenzidine and hydrogen peroxide) was added and the mixture incubated at room temperature for $30 \mathrm{~min}$. The enzyme reaction was stopped by the addition of $100 \mu \mathrm{l}$ of $1 \mathrm{M} \mathrm{HCl}$, the optical density was measured at $450 \mathrm{~nm}$ (with correction between 570 and $590 \mathrm{~nm}$ ) on a Dynatech Medicinal Products (Guernsey, Channel Island, U.K.) MX microplate reader, and the concentration of $\mathrm{p}$ JNK1/2 determined directly from a calibration curve generated using recombinant phosphorylated c-Jun$\mathrm{N}$-terminal protein kinase.

\section{Total JNK1/2}

Assays were performed using Total JNK1 Colorimetric (EIA) TiterZyme ${ }^{\circledR}$ Kits (Assay Designs; product number 900-107) employing a protocol that was essentially the same as that for $\mathrm{p}-\mathrm{JNK} 1 / 2$.

\section{Nitric oxide}

Assays were performed using Total Nitric Oxide Kits (Assay Designs; product number 917-020). Blood serum $(25 \mu \mathrm{l})$ was diluted with $25 \mu \mathrm{l}$ of 
HEPES buffer containing detergents and preservatives, and incubated with nitrate reductase in the presence of NADH for $30 \mathrm{~min}$ at $37^{\circ} \mathrm{C}$. The total nitrite formed was determined by Griess reaction with a solution of sulfanilamide and $\mathrm{N}$ (1-naphthyl)ethylenediamine in $2 \mathrm{M}$ hydrochloric acid in wells of a microtitre plate. After a $10 \mathrm{~min}$ incubation, the optical density of the colored azo-dye product was measured at $570 \mathrm{~nm}$ and the concentration of total NO determined directly from a calibration curve generated using nitrate standard.

\section{Cortisol}

Assays were performed using Cortisol Colorimetric (EIA) Correlate ${ }^{\circledR}$ Kits (Assay Designs; product number 900-0071) containing goat antibody specific to mouse IgG immobilized on a microtitre plate. Blood serum $(0.5 \mathrm{ml})$ was extracted twice with equal volumes of diethyl ether, the organic phases were separated, combined, evaporated to dryness under nitrogen, and the resulting residue stored in a freezer at $-20^{\circ} \mathrm{C}$. Frozen residues were dissolved in $0.5 \mathrm{ml}$ of assay buffer containing sodium azide immediately prior to assay. Test solutions, or reference standards, and mouse monoclonal antibody to cortisol were pipetted in duplicate into wells of the microtitre plate and incubated at room temperature for $1 \mathrm{~h}$. Alkaline phosphatase-cortisol EIA conjugate was added and, following a further incubation of $1 \mathrm{~h}$, excess reagents were washed out and the substrate solution ( $p$-nitrophenyl phosphate) added. After a further incubation of $1 \mathrm{~h}$, the enzyme reaction was stopped, the optical density measured at $405 \mathrm{~nm}$, and the concentration of cortisol determined directly from a calibration curve generated using cortisol standard.

\section{Testosterone}

Assays were performed using Testosterone Colorimetric (EIA) Correlate ${ }^{\circledR}$ Kits (Assay Designs; product number 900-065) employing a protocol that was essentially the same as that for cortisol.

\section{Leukotriene $\mathrm{B}_{4}$}

Assays were performed using Leukotriene $\mathrm{B}_{4}$ Colorimetric (EIA) Correlate ${ }^{\circledR}$ Kits (Assay Designs; product number 900-068) containing goat antibody specific to rabbit $\mathrm{IgG}$ immobilized on a microtitre plate. Blood serum $(0.5 \mathrm{ml})$ was acidified to $\mathrm{pH} 3.5$ by the addition of $25 \mu \mathrm{l}$ of $2 \mathrm{M}$ $\mathrm{HC} 1$, incubated at $4^{\circ} \mathrm{C}$ for $15 \mathrm{~min}$ and centrifuged at $2000 \mathrm{rpm}$ in a micro-centrifuge for $2 \mathrm{~min}$. The supernatant was separated and applied to a Supelco (Belefonte, PA, U.S.A.) Supelclean LC-18 SPE reverse phase cartridge that had previously been washed with $10 \mathrm{~mL}$ of ethanol and $10 \mathrm{~mL}$ of deionized water. The cartridge was eluted sequentially with $5 \mathrm{~mL}$ of water, $5 \mathrm{~mL}$ of $15 \%$ ethanol, $5 \mathrm{~mL}$ hexane and $5 \mathrm{~mL}$ ethyl acetate. The ethyl acetate fraction was evaporated to dryness on a rotary evaporator and dissolved in $500 \mu$ of Tris buffered saline containing proteins, detergents and sodium azide as preservative. Test solutions, suitably diluted with assay buffer, or reference standards, rabbit polyclonal antibody to leukotriene $\mathrm{B}_{4}$ and alkaline phosphatase-leukotriene $\mathrm{B}_{4}$ EIA conjugate were pipetted in duplicate into wells of the microtitre plate and incubated at room temperature for $2 \mathrm{~h}$. Excess reagents were washed out and the substrate solution ( $p$-nitrophenyl phosphate) added. After a further incubation of $2 \mathrm{~h}$, the enzyme reaction was stopped, the optical density measured at $405 \mathrm{~nm}$, and the concentration of leukotriene $\mathrm{B}_{4}$ determined directly from a calibration curve generated using leukotriene $\mathrm{B}_{4}$ standard.

\section{Prostaglandin $\mathrm{E}_{2}$ and Thromboxane $\mathrm{B}_{2}$} Assays were performed using Assay Design Colorimetric (EIA) Correlate ${ }^{\circledR}$ Kits for Prostaglandin $\mathrm{E}_{2}$ and Thromboxane $\mathrm{B}_{2}$ (product numbers 900001 and 900-002, respectively) employing protocols that were similar to that employed in the assay of leukotriene $\mathrm{B}_{4}$.

\section{Statistical analysis}

Data management and statistical analyses were performed using GraphPad (San Diego, CA, U.S.A.) Prism software (version 3.03 for Windows). The significance of between-group differences (at $95 \%$ confidence intervals) in the normalized mean values of analytes measured in blood plasma of rabbits were examined using two-tailed unpaired $t$-tests or Mann-Whitney tests; within-group comparisons were made using paired $t$-tests. The significance of the between-group differences in the normalized mean values of analytes measured on days 1 and 8 of treatment, and of those measured before and after stress, were determined using oneway ANOVA with Tukey's or Dunnett's multiplecomparison ad hoc tests. 


\section{Results}

Water (placebo group A) or rhodioloside or an extract of E. senticosus, $R$. rosea, $S$. chinensis, $B$. alba or $P$. ginseng (verum groups $B$ and $C$ ) was administered orally to rabbits each day (commencing on day 2) for 7 days. Ten minutes after the administration of drug or placebo, animals in groups A and $\mathrm{C}$ were subjected to $2 \mathrm{~h}$ of stress by immobilization on days 2 and 8; animals in group B were not subjected to stress. Blood samples were taken from all rabbits on days 1 and 8 , and also on day 2 for animals in groups B and C. Sampling on days 2 and 8 took place immediately after the application of stress (groups A and C) or at the same designated time (group B). Blood samples were analyzed for content of SAPK/JNK, p-SAPK/p$\mathrm{JNK}, \mathrm{NO}$, cortisol, testosterone, prostaglandin $\mathrm{E}_{2}$, leukotriene $\mathrm{B}_{4}$, and thromboxane $\mathrm{B}_{2}$.

The coefficients of variation in the levels of the assayed markers at the beginning of the study were found to vary from 2 to $100 \%$ (Table 2) even though the animals had all been kept under identical conditions. Hence all data were normalized separately for each rabbit with respect to the initial (day 1) level (taken as 100\%) such that analyte concentrations could be expressed as a percentage of this basal value.
Figure 1 shows the levels of assayed markers measured in the blood of placebo rabbits (group A) sampled immediately after immobilization stress applied on day 8 and expressed as percentages of the basal levels determined on day 1 of the study. Only the contents of p-SAPK/p-JNK, NO and cortisol were significantly increased (by between 200-300\% cf. to basal levels: Table 3) following the application of stress. In Fig. 2, the post-stress levels (expressed as percentages of basal levels) of p-SAPK/p-JNK, NO and cortisol measured on day 8 in the blood of verum group $C$ animals, who had received multiple doses of study drugs, are compared with those of the placebo group. It is clear that, following the repeated administration of adaptogens/stress-protectors, the levels of the stress markers NO and cortisol remained practically unchanged $(\mathrm{P}>0.05)$ from the basal values after the period of acute stress (Table 3 ). Rhodioloside and extracts of $S$. chinensis and $R$. rosea were the most active adaptogens with respect to their capability to inhibit $\mathrm{p}-\mathrm{SAPK} / \mathrm{p}-\mathrm{JNK}$ formation during stress.

The percentage changes in the blood levels of stress markers in verum group $\mathrm{C}$ animals after single (day 2) and multiple (day 8) administration of adaptogens, in comparison with those

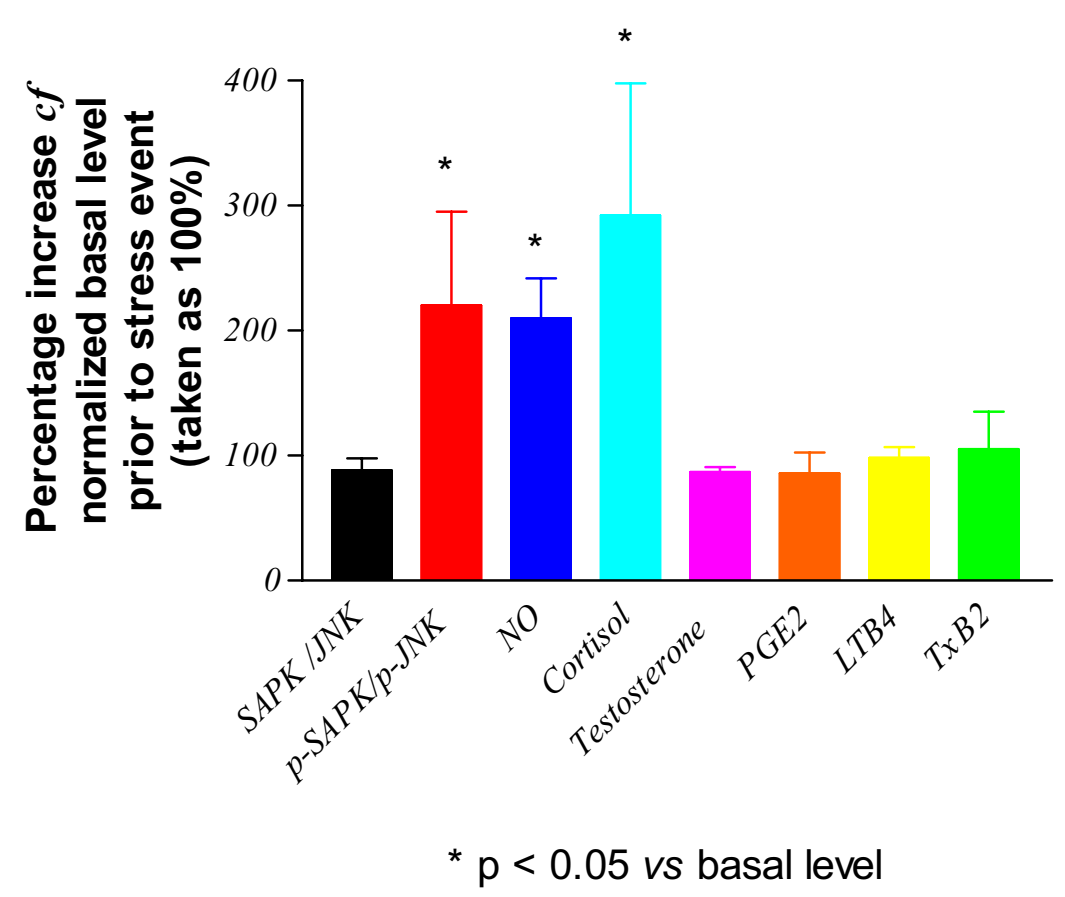

Figure 1. Stress-induced changes in the concentration of analytes in the blood of rabbits treated with placebo (group A). SAPK/JNK: stress-activated protein kinase; p-SAPK/JNK: phosphorylated-SAPK/JNK; NO: nitric oxide; PGE2: prostaglandin E2; LTB4: leukotriene B4; TxB2: thromboxane B2. 
Table 2. Mean basal levels of biochemical markers ${ }^{a}$ determined in rabbits on 1 day of the study prior to treatment or stress conditions.

\begin{tabular}{|c|c|c|c|c|c|c|c|c|}
\hline $\begin{array}{l}\text { Study } \\
\text { animal }\end{array}$ & $\begin{array}{l}\text { SAPK/JNK } \\
\text { (ng/ml) }\end{array}$ & $\begin{array}{c}\text { p-SAPK/p-JNK } \\
\text { (ng/ml) }\end{array}$ & $\begin{array}{c}\mathrm{NO} \\
\text { (nmol/ml) }\end{array}$ & $\begin{array}{l}\text { Cortisol } \\
\text { (pg/ml) }\end{array}$ & $\begin{array}{c}\text { Testosterone } \\
\text { (pg/ml) }\end{array}$ & $\begin{array}{l}\mathrm{PGE}_{2} \\
(\mathrm{pg} / \mathrm{cl})\end{array}$ & $\begin{array}{l}\mathrm{LTB}_{4} \\
(\mathrm{pg} / \mathrm{cl})\end{array}$ & $\begin{array}{c}\mathrm{TxB}_{2} \\
\text { (pg/cl) }\end{array}$ \\
\hline 1 & 1952 & 45 & 145 & 1767 & 1391 & 3844 & 4580 & 3346 \\
\hline 2 & 1832 & 59 & 133 & 2793 & 1117 & 3657 & 3568 & 5917 \\
\hline 3 & 2272 & 35 & 66 & 1657 & 1437 & 3332 & 4817 & 2446 \\
\hline 4 & 1517 & 45 & 106 & 1177 & 1362 & 3869 & 3278 & 8894 \\
\hline 5 & 3112 & 66 & 40 & 7456 & 653 & 3426 & 3198 & 8129 \\
\hline 6 & 1833 & 83 & 59 & 1642 & 1718 & 2276 & 3190 & 10728 \\
\hline 7 & 5558 & 118 & 99 & 2969 & 1857 & 1938 & 4161 & 7963 \\
\hline 8 & 1910 & 120 & 64 & 8017 & 1373 & 2276 & 2269 & 5098 \\
\hline 9 & 1378 & 93 & 216 & 2500 & 1791 & 1886 & 1451 & 5377 \\
\hline 10 & 1456 & 77 & 321 & 4549 & 1631 & 2731 & 3705 & 1673 \\
\hline 11 & 1376 & 185 & 173 & 4817 & 1185 & 2053 & 3854 & 5460 \\
\hline 12 & 2123 & 203 & 322 & 2597 & 1296 & 2676 & 4010 & 7971 \\
\hline 13 & 2595 & 291 & 95 & 8491 & 1472 & 1085 & 1840 & 4163 \\
\hline 14 & 1877 & 281 & 221 & 6708 & 1043 & 773 & 1783 & 7361 \\
\hline 15 & 1326 & 251 & 258 & 1218 & 1070 & 1847 & 4014 & 9825 \\
\hline 16 & 4164 & 48 & 48 & 1388 & 1552 & 3292 & 1700 & 3970 \\
\hline 17 & 802 & 20 & 37 & 2708 & 718 & 1551 & 3906 & 4830 \\
\hline 18 & 1758 & 53 & 45 & 6018 & 1726 & 1220 & 4588 & 2198 \\
\hline 19 & 4914 & 26 & 31 & 8206 & 1461 & 4068 & 3840 & 1014 \\
\hline 20 & 1832 & 10 & 30 & 8917 & 745 & 4054 & 3826 & 4340 \\
\hline 21 & 1614 & 43 & 324 & 10000 & 2554 & 1118 & 3650 & 1018 \\
\hline 22 & 1373 & 276 & 178 & 2169 & 428 & 2442 & 3362 & 9551 \\
\hline 23 & 1997 & 165 & 96 & 4315 & 1016 & 2161 & 3122 & 10040 \\
\hline 24 & 1407 & 175 & 56 & 9913 & 3132 & 1451 & 3049 & 10025 \\
\hline 25 & 5200 & 275 & 179 & 10000 & 730 & 1512 & 2113 & 1776 \\
\hline 26 & 1914 & 277 & 197 & 12700 & 1383 & 1481 & 3198 & 1604 \\
\hline 27 & 2109 & 209 & 61 & 12500 & 1608 & 991 & 3100 & 1624 \\
\hline 28 & 1717 & 36 & 56 & 1632 & 1002 & 3272 & 2949 & 3199 \\
\hline 29 & 1493 & 42 & 66 & 3710 & 735 & 4248 & 2758 & 3710 \\
\hline 30 & 1779 & 108 & 41 & 6020 & 1127 & 3878 & 2850 & 3121 \\
\hline 31 & 2807 & 63 & 214 & 4269 & 1219 & 2442 & 2953 & 9081 \\
\hline 32 & 1972 & 140 & 233 & 5267 & 1080 & 3033 & 2273 & 8597 \\
\hline 33 & 2007 & 167 & 176 & 7241 & 1506 & 2383 & 2454 & 7960 \\
\hline 34 & 2238 & 57 & 285 & 8974 & 1182 & 2677 & 3353 & 3303 \\
\hline 35 & 1324 & 129 & 25 & 9606 & 1053 & 4320 & 2582 & 9790 \\
\hline 36 & 1675 & 106 & 77 & 5469 & 1149 & 2730 & 1338 & 9382 \\
\hline 37 & 3550 & 212 & 95 & 5733 & 2464 & 2248 & 2871 & 1669 \\
\hline 38 & 2033 & 163 & 17 & 12600 & 1472 & 2690 & 2515 & 2839 \\
\hline 39 & 1346 & 245 & 43 & 6237 & 2301 & 3102 & 2328 & 2780 \\
\hline Mean & 2183.1 & 128.1 & 126.3 & 5742.2 & 1378.0 & 2564.9 & 3087.1 & 5430.0 \\
\hline SD & 1091 & 88 & 93 & 3448 & 547 & 1001 & 872 & 3152 \\
\hline CV\% & 50 & 68 & 74 & 60 & 40 & 39 & 28 & 58 \\
\hline Minimum & 802 & 10 & 17 & 1177 & 428 & 773 & 1338 & 1014 \\
\hline Maximum & 5558 & 291 & 324 & 12700 & 3132 & 4320 & 4817 & 10728 \\
\hline Range & 4757 & 280 & 308 & 11523 & 2704 & 3547 & 3479 & 9714 \\
\hline Min/Max & 7 & 28 & 20 & 11 & 7 & 6 & 4 & 11 \\
\hline
\end{tabular}

aSAPK/JNK: stress-activated protein kinase; p-SAPK/JNK: phosphorylated-SAPK/JNK; NO: nitric oxide;

$\mathrm{PGE}_{2}$ : prostaglandin $\mathrm{E}_{2} ; \mathrm{LTB}_{4}$ : leukotriene $\mathrm{B}_{4} ; \mathrm{TxB}_{2}$ : thromboxane $\mathrm{B}_{2}$. 
determined at the start of the study (day 1), are presented in Table 4. There were few significant changes in levels of the stress markers in resting animals after single or repeated administration of adaptogens. Thus, total JNK increased after single (but not multiple) application of $R$. rosea extract and its active component rhodioloside. It would appear that repeated treatment with these drugs results in adaptation of the organism to the "stressors". A single dose of rhodioloside or of an extract of $S$. chinensis or $R$. rosea decreased the thromboxane $\mathrm{B}_{2}$ level implying inhibition of platelet aggregation, blood clotting and an antistress effect. Testosterone significantly increased after repeated administration of an extract of $E$. senticosus, an effect that is consistent with previous observations (Winterhoff et al. 1993) also indicating an anti-stress effect of this drug

\section{Discussion}

The mechanisms of action of plant-derived stressprotectors and adaptogens, and the biochemical markers that need to be assayed in order to evaluate the efficacy of such drugs, have yet to be fully elucidated. Thus the aim of the present study was to develop a simple laboratory test for the evaluation of the efficacy of adaptogens. Typically, blood (serum or plasma) is the most common and convenient biological fluid for routine tests since it is not necessary to kill the animal and no special skills or facilities for surgery are required in order to obtain samples. Blood is the liquid that is in contact with all tissues, including the brain (where the most important regulatory processes associated with the effects of adaptogens take place), and provides a unique source in which all of the studied hormones, including cortisone, testosterone and thromboxane $\mathrm{B}_{2}$, can be measured. For these reasons, the experiments described in this paper were conducted using blood samples rather than brain tissues. The sources of JNK and p-JNK are blood plasma cells, presumably the PMNL and lymphocytes, which were subjected to lysis prior to assay. It is assumed that the nitric oxide originated both from blood cells and from many other tissues, including the brain.

In the present study, the blood levels of potential stress response markers were determined in laboratory rats in both the resting state and after restraint stress. The basis for choosing the specific stress markers assayed is described below.

\section{SAPK/JNK and $p-S A P K / p-J N K$ :}

These kinases belong to a family of enzymes (the mitogen-activated protein kinases, MAPK) that act within the signaling systems by which cells transduce extracellular stimuli into intracellular responses. Such signal transduction mediators are distributed extensively throughout the CNS and regulate a diverse array of cellular functions. The most common MAPKs are the extracellular signalregulated kinases that primarily regulate cellular

(a)

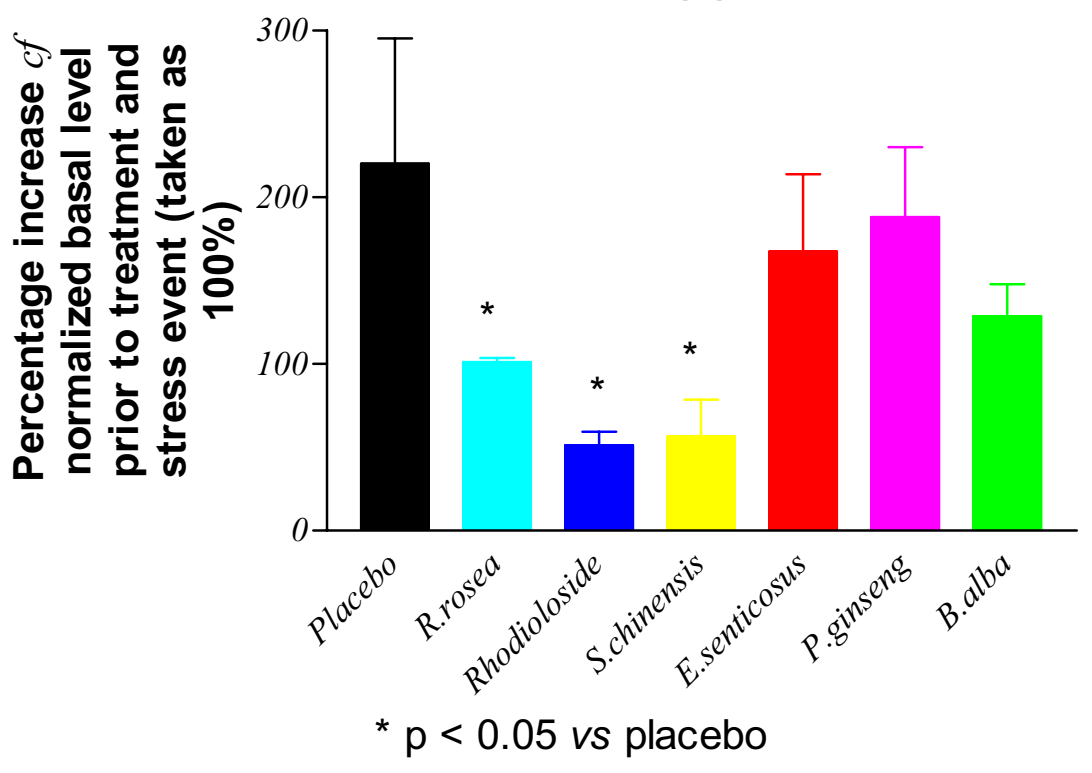




\section{(b)}

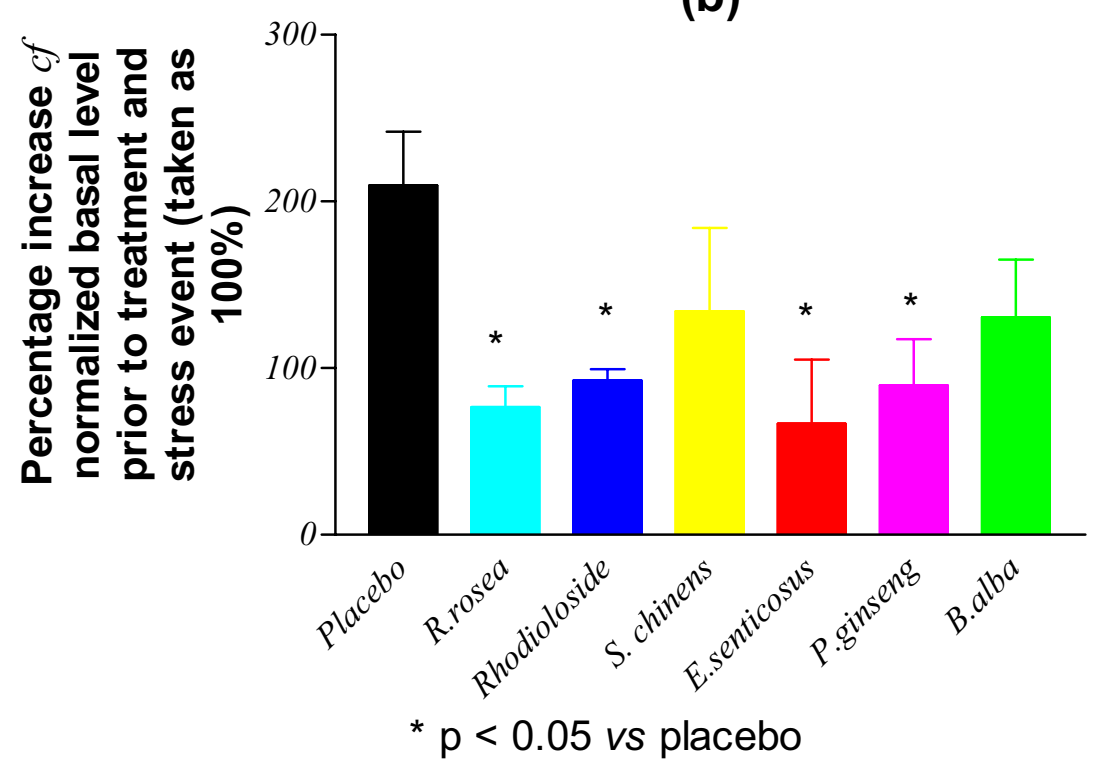

(c)

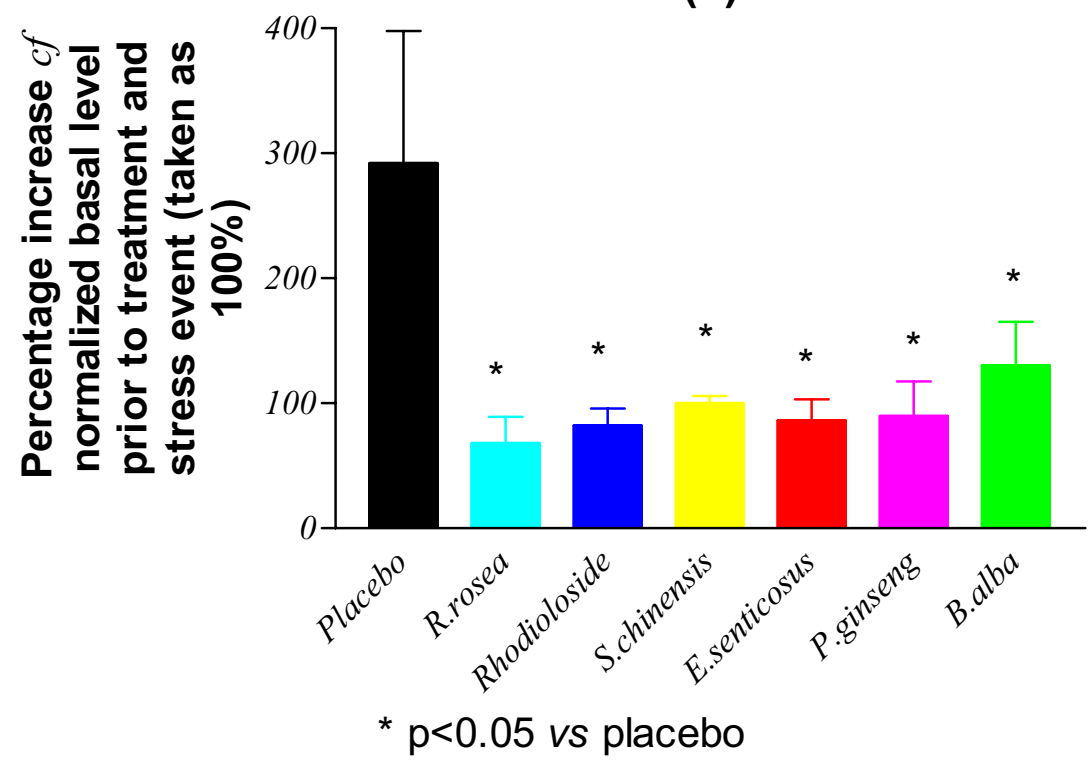

Figure 2. Stress-induced changes in the concentration of: (a) phosphorylated stress-activated protein kinase (p-SAPK/p-JNK), (b) nitric oxide and (c) cortisol in the blood of rabbits treated with a placebo or multiple doses of adaptogens/stress-protectors.

growth, differentiation, apoptosis, survival, differentiation and adaptation to stress (Schaeffer and Weber, 1999; Kyriakis and Avruch, 2001). SAPK/ JNK is activated by diverse stress and pro-inflammatory stimuli including cytokines, growth factors, irradiation, hyperosmolality, cold, heat and shear stress, muscle contraction and exercise (Williamson et al. 2003; Shen et al. 2004). The activation of SAPK/JNK occurs through phosphorylation at Thr183 and Tyr185, and the resulting phosphorylated kinase can translocate to the nucleus where it regulates transcription through its effects on c-Jun, activating transcription factor-2 (ATF-2) and activator protein 1 (AP-1) transcription factor. The prolonged activation of $\mathrm{JNK}$ and the subsequent phosphorylation of various transcription factors have been implicated in the initiation of the apoptosis cascade in some cell lines, and may represent the initiating factor in the pathogenesis of overuse injuries. Moreover, SAPK/JNK is believed to be important in neuronal development, CD4 T-helper-cell differentiation, T-cell activation, 


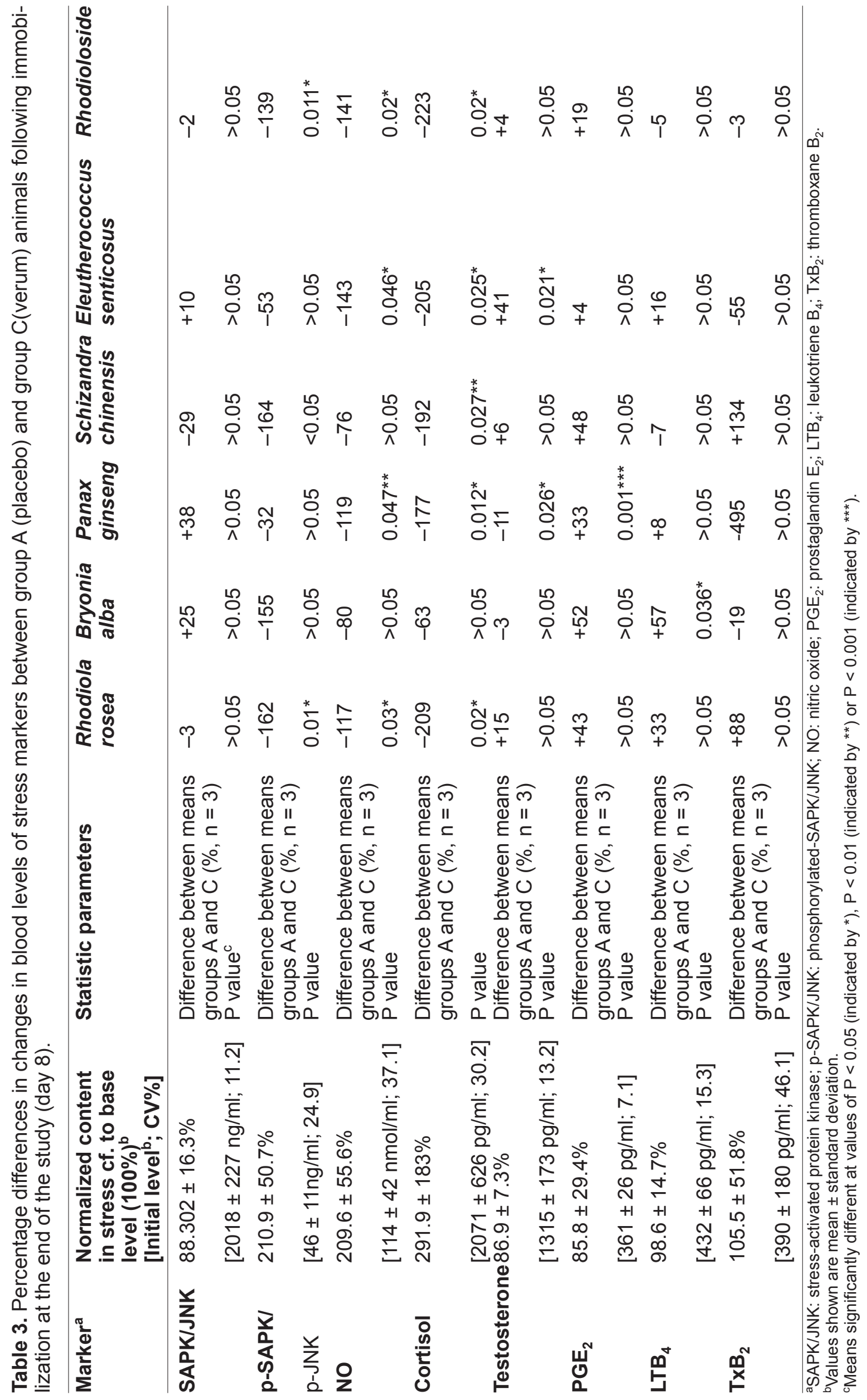




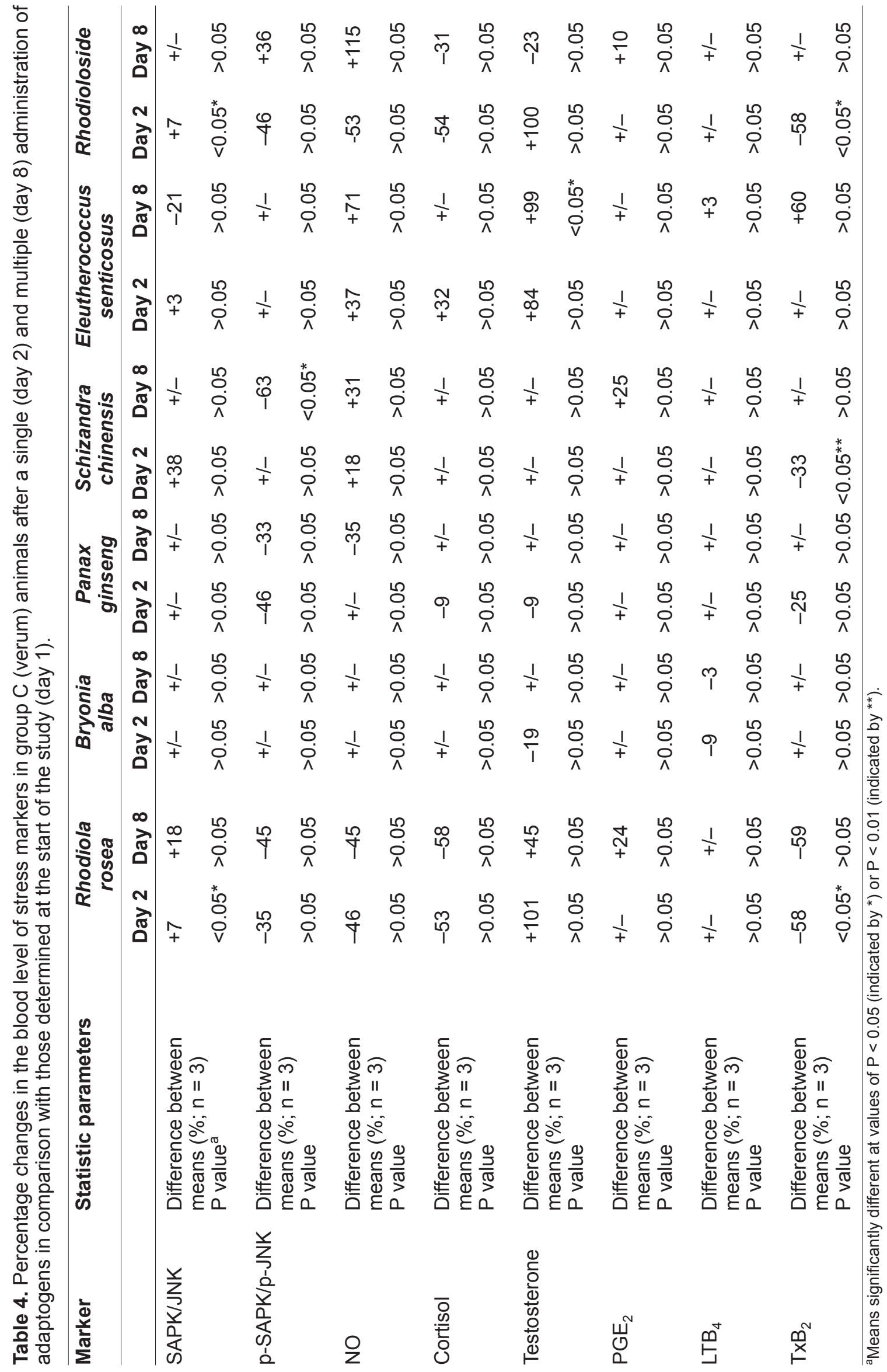


and the pro-apoptotic response to genotoxins (Kyriakis and Avruch, 2001).

\section{Nitric oxide}

$\mathrm{NO}$ is a short-lived free radical that can be produced in mammalian cells by a family of NO synthases (NOS), including neuronal (nNOS), endothelial (eNOS) and inducible (iNOS) enzymes, members of which have been shown to function as intracellular signaling regulators in a variety of cellular events (Nathan and Xie, 1994). While nNOS and eNOS are constitutively expressed and their enzymatic activities regulated by changes in intracellular concentrations of free $\mathrm{Ca}^{2+}$, iNOS is regulated at the transcriptional level. The expression of iNOS can be induced by cytokines and lipopolysaccharides in many cell types including macrophages. iNOS, which produces high levels of NO, plays a role in cellular immune responses (Nathan, 1997), exerting many of its functions, including signal transduction, DNA repair, host defense, blood pressure control, and neurotransmission, through $S$ nitrosylation of proteins (Stamler, 1994). Changes in gene expression and enzymatic activity of nNOS in the hypothalamus, pituitary and adrenal glands of rats subjected to immobilization stress have been reported (Liu et al. 1996). Furthermore, psychological and/or physiological stress causes NO release in, and may modulate stress-induced activation of, the HPA axis and the sympatho-adrenal medullary system (Kishimoto et al. 1996). Endogenous NO can suppress JNK/SAPK through a thiol-redox mechanism (Park et al. 2000).

\section{Cortisol}

This corticosteroid hormone is produced by the adrenal cortex and is known to be involved in the response to stress suppression in the immune system. Increased serum cortisol levels have been observed in connection with clinical depression and psychological stress involving stressors such as hypoglycemia, illness, fever, trauma, surgery, fear, pain, physical exertion or extremes of temperature. In normal release, cortisol has widespread actions that help restore homeostasis after stress. It acts as a physiological antagonist to insulin by promoting gluconeogenesis, breakdown of lipids and proteins, and mobilization of extrahepatic amino acids and ketone bodies. This leads to increased blood glucose concentrations, resulting in increased glycogen formation in the liver
(Freeman, 2005). In chronic stress, prolonged cortisol secretion causes muscle wastage, hyperglycemia, and suppresses immune/inflammatory responses. Moreover, long-term exposure to cortisol results in damage to cells of the hippocampus that may cause impaired learning. However, short-term exposure of cortisol helps to create memory, and constitutes the proposed mechanism for the storage of flash bulb memories.

\section{Testosterone}

The principal male sex hormone and an anabolic steroid that promotes cell growth and division resulting in the growth of several types of tissues, especially muscle and bone. Administration of testosterone produces numerous physiological effects including increased protein synthesis, muscle mass, strength, appetite, bone growth, increased libido, etc. Natural levels of testosterone decline gradually with age in men and also decrease during stress.

\section{Prostaglandin $E_{2}$, leukotriene $B_{4}$ and thromboxane A2}

These are eicosanoids that are known to play important roles in the early stages of stress response exerting both pro- and anti-inflammatory actions. These mediators are involved in the initiation, transmission and modulation of stress, as well as in the expression of stress symptoms. The synthesis of prostaglandins has been found to be stimulated by most stressors including heat pain, trauma, exercise, bacteria, restraint, water immersion, cage switching, examination, surgery, immobilization etc. The prostaglandins are known to activate the HPA axis through direct stimulation of corticotrophin releasing factor (CRF), vasopressin (AVP) neurons and noradrenergic neurons in the paraventricular nucleus (Fink, 2000).

Of the potential stress markers assayed in the present study, only p-SAPK/p-JNK, NO and cortisol were increased significantly above resting levels following application of immobilization stress to laboratory rabbits. However, after repeated administration of the adaptogens/stress-protectors, E. senticosus, R. rosea, S. chinensis, B. alba, $P$. ginseng and rhodioloside, the levels of NO and cortisol remained practically unchanged from normal during restraint stress.

The inhibition of stress-induced NO production demonstrated in this study is noteworthy since it may 
provide an explanation of the energy bursting effects of adaptogens in which the phase of endurance is prolonged and that of fatigue postponed. In this context, it is known that NO formation can strongly inhibit the production of cellular energy through two mechanisms: (i) inhibition of mitochondrial respiration by reversible (from constitutive isoforms of NOS) and irreversible (from iNOS) inhibition of cytochrome P450 (Brown, 2001), and (ii) the inhibition of glycolysis through modification of SH-groups of glyceraldehyde-3-phosphate dehydrogenase (Hara et al. 2006).

It has previously been reported that ginseng saponins act primarily on the hypothalamus and/or hypophysis producing a stimulation in the secretion of adrenocorticotrophic hormone (ACTH) within 30-90 min after single oral or intraperitoneal treatment, together with increased synthesis of corticosterone in the adrenal cortex and an increase in the concentration of corticosterone in the plasma (Hiai et al. 1979a, b; Filaretov et al. 1988). The mild stress-protective activity of the ginseng saponins is believed to be mediated through the blocking of ACTH action in the adrenal gland (Kim et al. 2003a, b) and by inducing NO production in the brain (Kim et al. 1998). Administration of a single dose of $P$. ginseng increased working capacity in rats by up to $132 \%$ (Filaretov et al. 1988): interestingly, although the effect of repeated administration over a 7 day period was more pronounced (179\%), it was not accompanied by further changes in blood cortisone level. Additionally, ginseng saponins have been shown to affect brain monoamine levels in heat-stressed mice causing a reduction in the stress-induced increase of noradrenalin and serotonin. Similar effects on the HPA axis under both normal and stress conditions were reported in experiments involving another tetracyclic triterpene glycoside, namely, cucurbitacin $\mathrm{R}$ diglucosides an active principle of $B$. alba, giving rise to a stress-protective and stimulating effect in animals (Panossian et al. 1997, 1999b).

The action of a steroid hormone is mainly attributed to its binding with a receptor, which results in the activation of the nuclear genome and subsequent alterations in protein synthesis. However, such a mechanism cannot account for the rapid effects of some adaptogens, particularly the single dose effects of $S$. chinensis and $R$. rosea (Panossian and Wagner, 2005). Moreover it has been shown that these adaptogens are able to enhance resistance in simple organisms, such as developing snail (Lymnaea stagnalis) embryos, silk worm (Bombix mori) larvae, round worms (Caenorhabditis elegans), and isolated cells (Reuber H35 hepatoma and isolated cardiac cells), against various conditions including cold-induced viral infection, Bacillus thuringiensis infection, stress induced by menadione, formalin, heat, and toxic metal ions (Chernykh et al. 1985; Boon-Niermeijer et al. 2000).

Such results clearly cannot be explained in terms of regulation of endocrine, immune or CNS systems, but rather confirm that adaptogens are universal enhancers of non-specific resistance of living organisms at various levels of organization, and that they can adapt cells and organisms to stress by mechanisms of regulation of intracellular communications. Whilst the effects of adaptogens on the HPA axis (Filaretov et al. 1986; Kimura and Sumiyoshi, 2004), on NO (Park et al. 1996; Panossian et al. 1999c) and eicosanoids (Panossian et al. 1988; Okhura et al. 1990; Park et al. 2005) are well documented, nothing is known about the involvement of SAPK/JPK, and its phosphorylated form, in the mechanism of action of plant adaptogens.

The results of the present study show that immobilization stress increases the levels of p-JNK in the blood cells of rabbits significantly $(\mathrm{P}<0.05)$ up to $200-300 \%$ compared with the initial basal level. Previously, a single 15 min session of forced swimming was found to increase $p-J N K$ ( $p$-JNK1 and/or p-JNK2/3) levels in all regions of the brain (hippocampus, neocortex, prefrontal cortex, amygdala and striatum) by ca 2-5-fold (Shen et al. 2004). Moreover, acute sessions of bicycle ergometry have been reported to produce increases in signaling intermediates from the SAPK/JNK pathways (Williamson et al. 2003). Interestingly, it was found that older men exhibited significantly $(\mathrm{P}<0.05)$ higher resting levels of $\mathrm{p}-\mathrm{SAPK} / \mathrm{p}-\mathrm{JNK}$ compared with younger men, but lower comparative levels after a session of the resistance exercise.

In the present study, the blood levels of $\mathrm{p}-\mathrm{SAPK} / \mathrm{p}-\mathrm{JNK}$ in rats that had been treated with multiple doses of the adaptogens $R$. rosea, $S$. chinensis and rhodioloside remained practically unchanged after the period of acute stress. These adaptogens contain mainly phenolic compounds and do not contain triterpenes. In contrast, repeated administration of stress-protectors that contained mainly triterpenes but no phenolics, produced little 
effect on $\mathrm{p}-\mathrm{SAPK} / \mathrm{p}-\mathrm{JNK}$ during stress. Under resting conditions, $R$. rosea and rhodioloside induced increases in total JNK after single (but not repeated) dose administration. It appears, therefore, that repeated administration of $R$. rosea and rhodioloside results in adaptation of the organism to these "stressors."

The curative effect of an extract of $S$. chinensis on patients with asthenia and depressive syndromes has been established in several studies (Staritsina, 1946; Zakharova, 1948; Leman, 1952). Furthermore, it has recently been demonstrated that the standardized extract SHR-5 from $R$. rosea possesses a clear and significant anti-depressive activity in patients suffering from mild to moderate depression (Darbinyan et al. 2006). It can be hypothesized that the antidepressant effects of $R$. rosea and $S$. chinensis are associated with the inhibition of emotional stress induced by the over-activation of $\mathrm{p}-\mathrm{SAPK} / \mathrm{p}-\mathrm{JNK}$. The SAPK/JNK pathway is known to be involved in the pathogenesis of glucocorticoid resistance (GR) found in certain chronic immune/inflammatory diseases and in subgroups of patients with major depression, and activation of SAPK/JNK has been reported to inhibit GR function (Wang et al. 2005). Moreover, physiologic activation of SAPK/JNK appears necessary for the induction of long term depression, and overactivation of these kinases by cytokines at pathophysiological concentrations is detrimental to long term potentiation. It has thus been suggested that SAPK/JNK pathways may represent a therapeutic target for the normalization of GR function in these disorders (Wang et al. 2005).

Since SAPK/JNK is activated in Alzheimer disease (Lagalwar et al. 2006), the inhibition of such activation might provide some protection from stress-induced apoptotic cell death. In this context, adaptogens could induce a positive effect in neurodegenerative disorders characterized by the loss of neurons in brain regions involved in learning and memory. Thus, it is suggested that the beneficial effects of $R$. rosea and rhodioloside on mental performance in stress, as well as the protection against neurotoxicity offered by $S$. chinensis, might be associated with their inhibitory effect on the formation of $\mathrm{p}-\mathrm{SAPK}$. Related data may be considered to add further support to the hypothesis that adaptogens have a therapeutic effect in neurological and neurodegenerative disorders. Thus, both $R$. rosea and rhodioloside inhibit propyl endoperoxidase, which is known to play a role in the degrada- tion of neuropeptides involved in the process of learning and memory (Fan et al. 2001). Moreover, rhodioloside may protect $\mathrm{PC} 12$ cells against the excitotoxic action of glutamate by suppressing the excessive entry of $\mathrm{Ca}^{2+}$ and the release of calcium stores (Cao et al. 2006), whilst an extract of $S$. chinensis fruit, and the active components schizandrins A, B and C, significantly reduce the neurotoxic action of glutamate (Kim et al. 2004).

\section{Conclusion}

It has been demonstrated that nitric acid and cortisol are appropriate stress markers that can be employed in the evaluation of the anti-stress effects of stress-protectors and adaptogens. It is noteworthy that only $\mathrm{p}-\mathrm{SAPK} / \mathrm{p}-\mathrm{JNK}$ appears to be a potential marker in bioassays of adaptogens and presumably of potential antidepressants.

\section{Notice of conflict of interest}

This study was funded with project grants from the Research and Development Division of the Swedish Herbal Institute (the Sponsor): all materials were supplied by the sponsor. The funding sponsor, however, had no role in any practical aspect of the study including experiments, data collection, management, analysis and interpretation of the data

The study was conceived by AP and the protocol of the study was formulated by AP. AH was responsible for experiments with animals and for the collection of samples for bioassays; MA performed the bioassays; AH and MA were involved in the data analysis, statistical evaluation and preparation of the draft report of the experimental part of the study. AP drafted the manuscript, and all authors (GW,AP, AH and $\mathrm{MH}$ ) were involved in its critical appraisal and final approval.

AP is employed by the Sponsor (SHI) on a permanent basis. MH has no commercial associations or financial interests with respect to this study: the work described forms part of her research project on adaptogens. AH receives an honorarium from the sponsor for contract research carried out on behalf of SHI.GW is the Director of Research and Development at SHI and is an SHI stockholder.

\section{References}

Aksenova, R.A., Zotova, M.I. and Nekhoda, MF. et al. 1968. Comparative characteristics of the stimulating and adaptogenic effects of Rhodiola rosea preparations. In Saratikov AS, ed. Stimulants of the central nervous system. Vol. 2. Tomsk:Tomsk University Press, p. 3-12. 
Boon-Niermeijer, E.K., van den, Berg, A. and Wikman, G. et al. 2000 Phytoadaptogens protect against environmental stress-induced death of embryos from the freshwater snail Lymnea stragnalis. Phytomedicine, 7:389-400.

Brekhman, II. and Dardymov, IV. 1968. New substances of plant origin which increase non-specific resistance. Ann. Rev. Pharmacol., 8:419-30.

Brown, G.C. 2001. Regulation of mitochondrial respiration by nitric oxide inhibition of cytochrome $c$ oxidase. Biochim Biophys Acta - Bioenergetics, 1504:46-57.

Cao, L.L., Du, G.H. and Wang, M.W. 2006. The effect of salidroside on cell damage induced by glutamate and intracellular free calcium in PC12 cells. J. Asian Nat. Prod. Res., 8:159-65.

Chernykh, S.I., Lukhtanov, V.A. and Simonenko, N.P. 1985. Adaptation to damage in the silkworm Bombyx mori L. (Lepidoptera bombycidae). III. Adaptogens and larval resistance to stress-induced activation of latent viral infection. Entomol. Obozrenye, 2:267-72.

Darbinyan, V., Aslanyan, G. and Amroyan, E. et al. 2006. Clinical trial of Rhodiola rosea L. extract SHR-5 in the treatment of mild to moderate depression. Nordic. J. Psychiatry, accepted for publication on 21 July 2006.

Fan, W., Tezuka, Y. and Ni, K.M. et al. 2001. Propyl endopeptidase inhibitors from the underground part of Rhodiola sachalinensis. Chem Pharm. Bull., 49:396-401.

Filaretov, A.A., Bogdanova, T.S. and Mitiushov, M.I. et al. 1986. Effect of adaptogens on the activity of the pituitary-adrenocortical system in rats. Biull. Eksp. Biol. Med., 101:573-4.

Filaretov, A.A., Bogdanova, T.S. and Podvigina T.T. et al. 1988. Role of pituitary-adrenocortical system in body adaptation abilities. Exp. Clin. Endocrinol., 92:129-36.

Fink, G. 2000. Encyclopaedia of stress. San Diego: Academic Press. $1-3$.

Freeman, S. 2005 . Biological science. 2nd ed. Englewood Cliffs: Pearson Prentice Hall.

Hara, M.R., Cascio, M.B. and Sawa, A. 2006. GAPDH as a sensor of NO stress. Biochim. Biophys. Acta., 1762:502-9.

Hiai, S., Yokoyama, H. and Oura, H. 1979a. Features of ginseng saponininduced corticosterone secretion. Endocrinol. Jpn., 26:737-40.

Hiai, S., Yokoyama, H. and Oura, H. et al. 1979b. Stimulation of pituitaryadrenocortical system by ginseng saponin. Endocrinol. Jpn., 26:661-5.

Kim, D.H., Jung, J.S. and Suh, H.W. et al. 1998. Inhibition of stressinduced plasma corticosterone levels by ginsenosides in mice: involvement of nitric oxide. Neuroreport, 9:2261-4.

Kim, D.H., Moon, Y.S. and Jung, J.S. et al. 2003a. Effects of ginseng saponin administered intraperitoneally on the hypothalamopituitary-adrenal axis in mice. Neurosci. Lett, 343:62-6.

Kim, D.H., Jung, J.S. and Moon, Y.S. et al. 2003b. Inhibition of intracerebroventricular injection stress-induced plasma corticosterone levels by intracerebroventricularly administered compound $\mathrm{K}$, a ginseng saponin metabolite, in mice. Biol. Pharm. Bull, 26:1035-8.

Kim, S.R., Lee, M.K., Koo and K.A. et al. 2004. Dibenzocyclooctadiene lignans from Schisandra chinensis protect primary cultures of rat cortical cells from glutamate-induced toxicity. J. Neurosci. Res., 76:397-405.

Kimura, Y. and Sumiyoshi, M. 2004. Effects of various Eleutherococcus senticosus cortex on swimming time, natural killer activity and corticosterone level in forced swimming stressed mice. J. Ethnopharmacol, 95:447-53.

Kishimoto, J., Tsuchiya, T. and Emson, P.C. et al. 1996. Immobilizationinduced stress activates neuronal nitric oxide synthase (nNOS) mRNA and protein in hypothalamic-pituitary-adrenal axis in rats. Brain Res., 720: 159-71.

Kyriakis, J.M. and Avruch, J. 2001. Mammalian mitogen-activated protein kinase pathways activated by stress and inflammation. Physiol. Rev., 81:807-69.
Lagalwar, S., Guillozet-Bongaarts, A.L. and Berry, R.W. et al. 2006 Formation of phospho-SAPK/JNK granules in the hippocampus is an early event in Alzheimer disease. J. Neuropathol. Exp. Neurol., 65:455-64.

Leman, M.F. 1952. Treatment of reactive and asthenic states of exogenous etiology using the far east Schizandra. J. Nevropatol. Psychiatr., 52: $67-70$.

Liu, J., Wang, X. and Shigenaga, M.K. et al. 1996. Immobilization stress causes oxidative damage to lipid, protein, and DNA in the brain of rats. FASEB J., 10:1532-8.

Lüllmann, H., Mohr, K. and Hein, L. et al. 2005. Color atlas of pharmacology. 3rd ed. Stuttgart: Georg Thieme Verlag.

Nathan, C. 1997. Inducible nitric oxide synthase: what difference does it make? J. Clin. Invest., 100:2417-23.

Nathan, C. and Xie, Q.W. 1994. Nitric oxide synthases: roles, tolls, and controls. Cell, 78:915-8.

Ohkura, Y., Mizoguchi, Y. and Morisawa, S. et al. 1990. Effect of gomisin A (TJN-101) on arachidonic acid cascade in macrophages. Jpn. J. Pharmacol., 52:331-6.

Panossian, A. 2003. Adaptogens: Tonic herbs for fatigue and stress. Alt Comp. Therap., 9:327-32.

Panossian, A. and Wagner, H. 2005. Stimulating effects of adaptogens: An overview of clinical trials of adaptogens with particular reference to their efficacy on single dose administration. Phytother. Res., 19:819-38.

Panossian, A. and Wikman, G. 2005. Effect of adaptogens on the central nervous system. Arq. Bras. Fitomed. Cient., 2:108-30.

Panossian, A.G., Sprygin, V.G., Dardimov, IV. 1988. Influence of Eleutherococcus and eleutherosides A, B, C, D and E on the arachidonic acid release and metabolism. Chem. Pharm. J., 7:776-9.

Panossian, A., Gabrielian, E. and Wagner, H. 1997. Plant adaptogens II. Bryonia as an adaptogen. Phytomedicine, 4:83-97.

Panossian, A.,Wikman, G. and Wagner, H. 1999a. Plant adaptogens III. Earlier and more recent aspects and concepts on their mode of action. Phytomedicine, 6, 287-300.

Panossian, A., Gabrielian, E. and Wagner, H. 1999b. On the mechanism of action of plant adaptogens with particular references on cucurbitacin R diglucoside. Phytomedicine, 6:147-55.

Panossian, A.G., Oganessian, A.S. and Ambartsumian, M. et al. 1999c. Effects of heavy physical exercise and adaptogens on nitric oxide content in human saliva. Phytomedicine, 6:17-26.

Park, Y.C., Lee, C.H. and Kang, H.S. et al. 1996. Ginsenoside-Rh1 and $\mathrm{Rh} 2$ inhibit the induction of nitric oxide synthesis in murine peritoneal macrophages. Biochem. Mol. Biol. Int., 40:751-7.

Park, H-S., Huh, S-H. and Kim, M-S. et al. 2000. Nitric oxide negatively regulates c-Jun $\mathrm{N}$-terminal kinase/stress-activated protein kinase by means of $S$-nitrosylation. Proc. Natl. Acad. Sci. U.S.A., 97:14382-7.

Park, E.K., Shin, Y.W. and Lee, H.U. et al. 2005. Inhibitory effect of ginsenoside $\mathrm{Rb} 1$ and compound $\mathrm{K}$ on $\mathrm{NO}$ and prostaglandin $\mathrm{E} 2$ biosyntheses of RAW264.7 cells induced by lipopolysaccharide. Biol. Pharm. Bull, 28:652-6.

Saratikov, A.S. and Krasnov, E.A. 2004. Rhodiola rosea (Golden root): a valuable medicinal plant. Tomsk: Tomsk University Press. p 1205.

Schaeffer, H.J. and Weber, M.J. 1999. Mitogen-activated protein kinases: Specific messages from ubiquitous messengers. Mol. Cell. Biol., 19:2435-44.

Shen, C., Tsimberg, Y. and Salvadore, C. et al. 2004. Activation of Erk and JNK MAPK pathways by acute swim stress in rat brain regions. BMC Neuroscience, 5:36.

Stamler, J.S. 1994. Redox signaling: nitrosylation and related target interactions of nitric oxide. Cell, 78:931-6.

Staritsina, ON. 1946. The experience of using Schizandra preparations against depressive states in psychiatry. Zdravookhr Kazakhstana, $6-7: 42-4$. 
Upton, R. 1999. Schisandra berry. Schisandra chinensis. Analytical, quality control and therapeutic monograph. American Herbal Pharmacopoea and Therapeutic Compendium. 1-26.

Wagner, H., Norr, H. and Winterhoff, H. 1994. Plant adaptogens. Phytomedicine, 1:63-76.

Wagner, H., Bauer, R. and Peigen, X. et al. 1996. Fructus Schisandrae. Chinese drug monographs and analysis: Radix Bupleuri (chaihu). Verlag für Ganzheit Medizin Koetzting/Bayer, 4:1-8.

Wang, X., Wu, H., Lakdawala, V.S. and Hu, F. et al. 2005. Inhibition of Jun N-terminal kinase (JNK) enhances glucocorticoid receptormediated function in mouse hippocampal HT22 cells. Neuropsychopharmacology, 30:242-9.

Williamson, D., Gallagher, P. and Harber, M. et al. 2003. Mitogenactivated protein kinase (MAPK) pathway activation: effects of age and acute exercise on human skeletal muscle. J. Physiol., 547:97787.
Winterhoff, H., Gumbinger, H.G. and Vahlensieck, U. et al. 1993. Effects of Eleutherococcus senticosus on the pituitary-adrenal system of rats. Pharmacol Lett., 3:99-102.

World Health Organisation. 1999. WHO Monographs on Selected Medicinal Plants. Vol 1. Geneva: WHO.

World Health Organisation. 2002. WHO Monographs on Selected Medicinal Plants. Vol 2. Geneva: WHO.

Zakharova PM. 1948. The experience of using Schizandra chinensis in psychiatric practice. In Krasnushkina EK, ed. Problems of social and clinical psychiatry. Vol. 9. Moscow: Moscow Regional NeuroPsychiatric Clinic. 271-278. 